\title{
Wireless network sensors that are energy efficient for monitoring and early warning
}

\author{
V. Ferrara \\ Department of Information Engineering, Electronics and \\ Telecommunications, Sapienza University of Rome, Italy
}

\begin{abstract}
Wireless sensor networks can be a convenient monitoring system for wide areas, because they are economically advantageous and they should be more specialized than remote sensing. Many different systems have been proposed as wireless sensor networks. Some networks are based on an IP protocol, as a web of objects. However, other solutions can be adopted when mostly the network is realized outside of an internet framework or when the internet connection can be limited at a few nodes. Architectures based on ZigBee protocol are an evident example. The IEEE 802.15.4 standard communication protocol of Wireless Personal Area Network (WPAN) defines the best characteristic of low-power, but limited data rate. It is a good candidate as the standard communication for many applications and monitoring activities. After the introduction of trends in the field of low power sensors networks, the design of a particular monitoring network will be presented: the early-warning of flood river control and intervention management.
\end{abstract}

Keywords: WSN, low power, low voltage.

\section{Introduction}

Disaster management needs a detailed monitoring of the environment, theatre of calamitous events, together with the actual human and material resources that are ready for intervention. Monitoring can be realized in different modes. For wide areas, as a country or province, remote sensing is more convenient. For non large terrestrial surfaces, distributed sensors are cheaper and more specialized. In the case of sensors at ground, they must detect both parameters of interest and the terrestrial coordinates where measurement was carried out. If it mounts a sensor 
in a mobile structure, this must include a GNSS (Global Navigation Satellite System). In some case, coordinates of relative or differential localization should be transmitted. If a sensor is fixed in a known position, its terrestrial coordinates are associated with the identification number of sensor. Distributed on the territory, the totality of sensors realizes a network. If the link is wireless, the network is a Wireless Sensors Network (WSN). Design of like network depends from area extension, maximum distance of link permitted from standard communication, maximum number of nodes, economical convenience and budget. After the introduction of trends in the field of sensors networks, the design of a particular monitoring network will be presented: early-warning of flood river control and intervention management. The aim of the article is to put in evidence advantages of sensor network in terms of detection specificity, maintenance, reduced consumption, energetic autonomy. The last ones are characteristics that derive from electronic technology development: they augment the freedom degrees in the position of nodes. It must be adopted harvesting energetic sources that are efficient. Many and different are the problems that must be resolved and challenges faced, but performances of designed system are promising, in terms of specialized monitoring, integration with other monitoring forms in a wider and complete management system and competitive cost.

\section{Sensor networks}

Monitoring of a wide geographical area can be efficiently obtained by remote sensing. This is a consequence of the high altitudes of satellite orbits which allows links to the most remote and least accessible areas. Even satellites with low earth orbit (LEO), that move at altitudes within 160 and $1600 \mathrm{~km}$ (below radiation belts, the named Van Allen belts), observe a large territory in any case. Other than geostationary orbit, the elliptical orbits are covered in a rotation time that depends from the altitude. So, monitoring continuity is not satisfied introducing a temporal resolution of satellite as time duration between two observations of the same area. Temporal resolution varies from hours to days and it can be the real limit of this monitoring methodology, in special mode when scenarios are that of emergency management. Satellite orbit, together with sensor characteristics determine the spatial resolution of the detection, like the amount of area covered by each pixel of the picture. Obviously, low orbits allow higher resolution.

In order to discover and to identify: elements, objects, materials, substances at ground, adequate spectral resolution must be used for sensing of sufficient range of the electromagnetic spectrum. At the same mode it need a suitable value of the radiometric resolution, i.e. the sensitivity for recording variations in the electromagnetic spectrum, measured in number of bits.

Alternative detections of data, complementary to remote sensing in some cases, and essentials for calibration of same remote sensing, could be realized. In effect, a network of at ground sensors constitutes valid system, when the 
continuity of a specific territorial area monitoring is the fundamental characteristic $[14,15]$.

We refer to a monitoring system that includes nodes and communication lines. One special node is the main node: the command and control station, the headquarters, where all data join from each remote node. In a complete form, nodes collect: sensors or actuators, communication device, power source and electronic digital management system as a microcontroller. Each node manages four phases: detection, data elaboration, transmission and reception of external inputs. In some cases a node can be only of re-transmission: it is missing of sensor unity and absolves the function of communication transit node. All nodes and communication lines together account for a network. Its structure is meshed preferably: each node communicates with other node different from main node normally. So, data is arriving to the main node from a remote node along a path that crosses other nodes. If each node is connected with all other nodes, the network is named completely connected. But increase of cost orients to realize a mesh network no completely connected in general.

The communication link can be wire type, but wireless technology is more convenient in the case of territorial area monitoring activity.

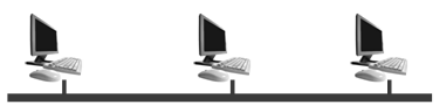

a)

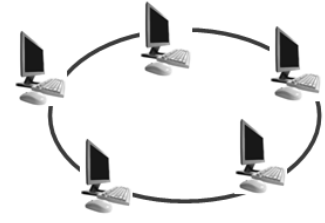

b)

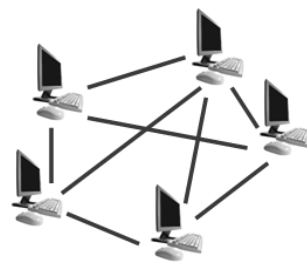

c)

Figure 1: Some network topologies: a) bus, used e.g. in the communication standard 802.3 (Ethernet wired); b) ring, adopted in the standards 802.5, FDDI, RPR 802.17; c) mesh no completely connected.

Generally, at ground, sensor networks cover a more limited area than that observed from a satellite. The reason resides in the number of nodes that increases considerably with the area augmentation. But low cost of single node design (it foresees more reduction in the near future) and of its maintenance allows optimization of network. At the present, nodes can be built in more small dimensions involving that device with very low power consumption. This feature, the technology of effective communication and increased performance computing, opens up new prospects for innovative architectures for the control and monitoring. In particular, the low power feature allows you to design your own power source based on the method of harvesting [9-11]. So, remote sensor location is independent by public power line and topology of mesh design can be defined by actual requirement of measure and by radio link only.

In any case, each node is identified univocally in the mesh. Its location is an element of identification, whereas measured quantity can be the same for different nodes. Generally it used a number identifying single node. This is 
similar at internet applications, where node identification is based on Internet Protocol (IP).

Many different systems have been proposed as wireless sensors networks. Moreover, operating area dimensions and size of devices classify the systems as: ubiquitous sensor networks, smart dust, smart objects.

\subsection{Smart dust}

Smart dust [1] is composed of large number of tiny electronic systems that measure the quantities as temperature and humidity over an area by use of spreading mode. Dispersion of these tiny unities in the area can be realized by using air flow for example. At the moment limitations for smart dust are in the communication method and computational capability.

\subsection{Smart objects}

The specificity of smart objects it is pointed out by different synonyms with which are named: web of objects, web of things, Internet of Things, cooperating objects. They are node in a web context. In any case a smart object includes: sensors and actuators interacting with the physical world, microprocessor which elaborates data collected from the sensors, communication devices for transmitting detected data and for receiving input from other smart objects and finally power source that provides the electrical energy.

Many are their applications: analysis of complex properties of air pollution, more simple parameter detections as temperature inside to containers, detection in the car parking, etc. Web service technology is used for this activity of monitoring. Web services are defined as communication between business servers. In the other words, at web site end, a user activates the web service functionality of smart objects, without requiring translation. It invokes a web service that does not reside in the World Wide Web of the user. In fact, web services provide an intermediary that allows travel agent server and another server to exchange data in owner framework. In order to realize a web service built on mechanisms that require communication bandwidth and processing power lower, a protocol more simple than SOAP (Simple Object Access Protocol) should be adopted. An alternative for smart objects is the Representational State Transfer (REST): a more lightweight architectural model which describes applications distribution. When data, like humidity, are transferred from server to client need only the knowledge of data representation (e.g. decimal representation) and the server-client connection. More information makes the model heavy, with consequences on communication bandwidth and power consumption.

An example of web service system for smart objects has been realized by Pachube web site. It is a system RESTfull, carried out around REST architecture. Users can submit data of sensors in a network on Pachube server. Moreover they can store and upload the same data. In order to use Pachube web site as an intermediary, company provides Application Program Interface (API) open, based on RESTfull model. Server absolves requests by means of HTTP protocol 
and formulates transfer of data by means a custom version of XML markup language, the Extended Environments Markup Language (EEML), or equivalent JSON or the more simple RSS or ATOM. Peculiarity of these markup languages is the inclusion in the tags of "environmental" information that are associated with the data: location coordinates, range of data values, unit of measure.

It can use a different technology of communication, but conveniently it should be based on Internet Protocol [2]. The choice of communication standard depends from many factors, whose one is the network extension (WLAN, WPAN, respectively wireless local area network and wireless personal area network). In case of smart objects a fundamental feature is low-power consumption. So, it is convenient to use IEEE 802.15.4, standard communication protocol of WPAN $[6,7]$, with best characteristic of low-power, but limited data rate: $250 \mathrm{Kbits} / \mathrm{s}$. Some studies have been realized for this standard communication, implemented on specific devices in order to assess the increase in power consumption [3,4]. Normally, this augmentation depends from the state and from the transition between two states.

Some specificities of smart objects make you want to use IP in open and interoperable form. So, companies in the fields of telecommunications, automation systems, etc., like Cisco, Ericsson, Atmel, Sun Microsystems, Dust networks and others, have founded in September 2008 an alliance to promote and to develop IP for Smart Objects: the IPSO alliance [5]. From initial 27 founding members, the alliance counts now about 50 members.

\subsubsection{ZigBee}

In any case, the identification by IP address of each network node is not the only way. Obviously, this is the choice in an immediate and organic web context.

Other solutions can be adopted, when mostly the network is realized outside of internet framework or when the internet connection can be limited at few nodes. In the last case, only these nodes will have an interface towards internet world. For many applications WPAN and low power IP architecture is evaluated too excessively with, no advantage. Consequently, there are architectures, like ZigBee, based on protocols that don't use IP.

ZigBee is a communication system, a network layer on top of the IEEE 802.15.4 standard, which includes PHYsical (PHY) and Message Authentication Code (MAC) layers. Transmission bands are in ISM (Industrial, Scientific and

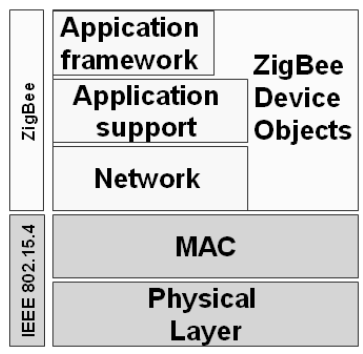

Figure 2: $\quad$ ZigBee protocol stack. 
Medical) band: $2.4 \mathrm{GHz}$, for international applications with $250 \mathrm{Kbits} / \mathrm{s}$ maximum throughput, $915 \mathrm{MHz}$, adopted in USA with maximum throughput of $40 \mathrm{Kbits} / \mathrm{s}$ and $868 \mathrm{MHz}$, in Europe at $20 \mathrm{Kbits} / \mathrm{s}$ bit-rate. Modulation is BiPhase Shift Keying (BPSK) for bands 868 and $915 \mathrm{MHz}$ and Offset quadrature phase-shift keying (OQPSK) for that at $2.4 \mathrm{GHz}$. Access mode is the Carrier Sense Multiple Access/Collision Avoidance (CSMA/CA): nodes occur if channel is free before transmission. But when transmission needs a low latency, the GTS (Guaranteed Time Slots) is the access mode.

Figure 2 shows its protocol stack. In particular the network layer is equivalent to IP layer of the architectures based on IP. At this layer competes routing and addressing. Application support layer is equivalent to the transport layer of IP architectures. There are three different ZigBee devices: Pan coordinator, router and End Device. Pan coordinator is one per network: it manages all nodes and it bootstraps the network. Router is an intermediate device that communicates with another node. Instead pan coordinator and routers are FFDs (Full-Function Device). End Device is a RFD: Reduced Function Device, showing lower functionality than coordinator and router. End devices communicate only with their router. Figure 3 shows classical topologies that can be implemented for the network: star and mesh. But, when two nodes must be connected between them, a more simple peer-to-peer topology could be realized.

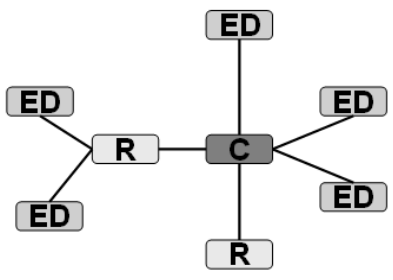

a)

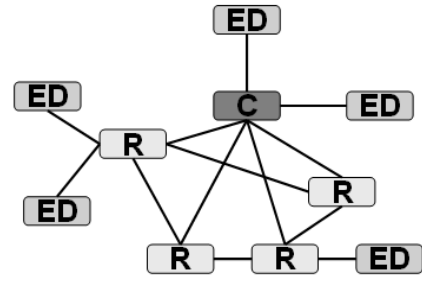

b)

Figure 3: Usual network topologies for ZigBee: a) star; b) mesh. Nodes ED, $\mathrm{R}$ and $\mathrm{C}$ denote respectively: End Devices, Router and Coordinator.

ZigBee transmits in two different modes: broadcast and unicast. The broadcast method sends data packet toward all nodes with expensive procedure. In unicast mode packets are sent only to the addressed node.

A commercial project which uses ZigBee protocol requires that the company participates, as membership, to Zigbee alliance, whereas for non-commercial projects the specifications can be downloaded from Zigbee web site [8]. In any case a custom protocol can be implemented on these transmitter-receiver devices. Custom design solution should be adopted when there are conveniences in terms of power consumption and loss. Alliances, like IPSO and ZigBee, are advantageous for interoperability and support, but they impose dependencies often: users are constrained to revise devices for innovating release, developed from the main companies of alliance. 


\section{Case study: river monitoring}

In order to control the flood of a river, we designed a monitoring system based on ground sensors network. Its main function is as an early warning in the case of an incipient flood event. As the smart objects, our network nodes include: sensors, microcontroller, transceiver and power supply.

Functions of a node are: measurement of environmental parameters (as temperature, humidity, level of river, detection of images), data elaboration, automatic transmission of eventual early warning, image transmission if requested. Actually, we provided in the prototype the implementation of image acquisition and its local elaboration for evaluating early warning. Essential components of a node are: PIC32MX360F512L microcontroller, MRF24J40MA IEEE 802.15.4 standard compliant radio frequency transceiver, OV7710 CMOS VGA colour CameraChip ${ }^{\mathrm{TM}}$. Microcontroller and transceiver are Microchip devices. The single-chip video/imaging camera device is an Omnivision image sensor. MRF24J40MA is a PCB module (Printed Circuit Board) and its dimensions are $1.1 \times 0.7 \times 0.03$ inches. It includes an integrated PCB antenna that has need an area around 1.2 inches be kept clear of metal objects. Transceiver module interfaces PIC microcontroller via 4-wire serial SPI interface.

The optical control of a target, one per node, allows automatic evaluation of flood warning condition. This method can be coupled with detection of surface water level of river that uses e.g. laser based system. OV7710 device can acquire also panoramic image. This one will be transmitted toward control centre if coordinator requires it. Low rate of IEEE 802.15.4-2003 standard limits the transmission of total image to on-demand only. Moreover the re-transmission along the nodes of the chain could determine reception delay. This delay can be decreased by imposing alternative standard communication. The number of these different transmitters must be evaluated when you define operative aspects of design.

Monitoring system will be organized by installing a network of these devices along the course of river. The distance between two nodes depends from sensitivity and output power of transceiver. There are transceivers that allow radio connection at one kilometre of distance. The greater is the distance the higher is energetic consumption of node. In order to obtain minimum number of energetically autonomous nodes, it is necessary a compromise. In our prototype design, transceiver covers about 100 meter of distance, but we collocate nodes about at 30-50 meter between them. This is allowed because the production cost of single node can be very low. River monitoring network topology, shown in figure 4 , assumes a line form. If other nodes, no-contiguous, are in visibility, jump is permitted in the case communication link at node fails.

Three protocol stacks are proposed from the transceiver for using in a specific network configuration: MiWi Peer-to-Peer, MiWi and ZigBee. In the case of a simple communication, with a couple of nodes, MiWi Peer-to-Peer is the protocol stack more convenient. For MiWi protocol stack, all nodes use the same multiple access in the channel. ZigBee protocol stack is more complete and complex among three protocols. 


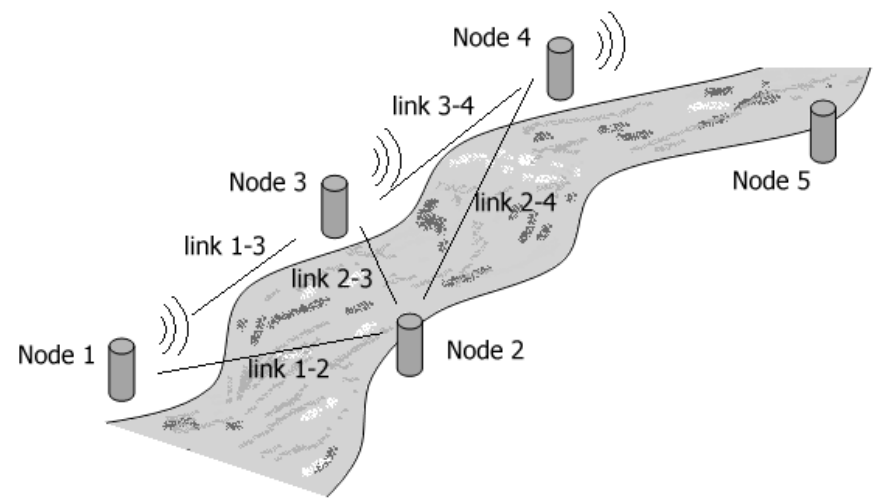

Figure 4: Topology of network is a simplified mesh. Normally a linear radio communication link connects progressively the nodes 1-2, 2-3, etc. But if a link fails, alternative connections are permitted.

In order to decrease energetic consumption of each node, a custom protocol has been used for this simplified mesh network.

At start, coordinator node evaluates requests of all nodes and assigns PAN ID: 16 bits identification number that defines the RF channel used from the network. After the start phase, coordinator node is placed in the LOCK state and network configuration is stored in a non-volatile memory. Reset of system can be realized by three different ways: soft reset, half reset and full reset. By using of soft reset system restart from statements saved in the memory. If it imposes half reset, all data are cancelled except for PAN ID that assures same nodes configuration. Finally, full reset starts a new network re-assignation. Coordinator checks the RF channel, considering communication noise also. Minimum test time is about ten seconds. After check, the coordinator starts half reset. Single node can require a channel hop, among 15 channels that are allowed, with each two between them $5 \mathrm{MHz}$ distant. This procedure is several minutes long, so it's convenient not to enable any node in this phase. Obviously, routing tables are dynamics, but for the particular mesh, memorization of all addresses can be avoided. In effect, it uses a sequential numeration: from 00:00 (exadecimal number) to maximum FF:FF. Number 00:00 is for MAC address of coordinator, whereas the progressive identification numbers are assigned to the other end devices.

In the case of mesh networks, the access mode CSMA/CA of the transceiver can be fulfilled by means of two different mechanisms: beacon and no-beacon. If no-beacon method has been used, all nodes can transmit only when state of channel is idle. An example is the MiWi protocol which uses no-beacon network. In the beacon method, node can transmit at pre-defined time ranges: the coordinator starts a beacon frame whose all nodes are synchronized. Normally, each node transmits and receives in its assigned time interval, inside the beacon frame. 
OV7710 CMOS sensor shows a VGA default resolution, size of active image array of 640columns per 480 rows (307,200 pixels) and transfer rate of 30 frame per second. Output formats are: $\mathrm{YCrCb} / \mathrm{YUV}$ 4:2:2, RGB raw, and finally 10 bits digital video. Since the microcontroller elaborates images data for detecting edges and contours, we choice RGB raw output format. Sensor converts light intensity (photons number) to proportional electrical charge. It is not sensible to light wavelength. So, filters (red, blue and green) must be placed before each pixel. The order of the matrix sequence defines the named Bayer matrix with $50 \%$ of green colour, $25 \%$ of red and $25 \%$ blue. Simple algorithms convert data from RGB raw to RGB and then to grey levels. The grey level format is introduced in order to apply edge detection algorithm $[12,13]$.

The control of output format and other controls as white balance and exposure time, can be realized by use of Serial Camera Control Bus (SCCB) and by configuring sensor registries from PIC32. Microcontroller communicates to optical sensor by means of two interfaces: the previous SCCB adopting I2C standard protocol and the first synchronous serial SPI interface for transmitting RGB raw data. The second serial SPI interface has been used for the communication with the transceiver.

In order to decrease energetic consumption some features are carried out. Specifically, a custom protocol is optimized to decrease re-mapping computational cost and latency. Moreover, by means of Clear Channel Assessment (CCA), MRF24J40MA transceiver can recognize if the channel is busy or idle. Three different modes are available for the recognition, respectively: if received energy exceeds a prefixed threshold, if there is a standard IEEE 802.15.4 signal without threshold constraints and finally if there is a standard IEEE 802.15.4 signal that exceeds a prefixed threshold. In particular, power intensity received is recorded into RSSI register. These modes allow one to adjust the power with which the transceiver is transmitting. By means of a Command Frame some test packets are sent together with request of an ACK message. Test packets are written on the records. Next they are compared with data of RSSI for usage of minimum transmission power. Finally, we have taken care of the management of sleep mode by means of essential temporization and duration of detection and elaboration activities. In effect, PIC32 microcontroller could absorb $300 \mathrm{~mA}$ of maximum electrical current intensity. Transceiver absorbs $23 \mathrm{~mA}$ if it transmits around to 100 meters, whereas absorption decreases to $2 \mu \mathrm{A}$ in sleep mode. These absorption values are evaluates with $3.3 \mathrm{~V}$ voltage supply for all devices. The routers are always active. The coordinator can be deactivated if it manages a mesh and no enddevice is connected to it. End-device; the device remains in sleep mode when not transmitting data and processes.

\section{References}

[1] J.M., R. Katz, K. Pister, Next century challenges: mobile networking for "smart dust", MobiCom '99: Proceedings of the $5^{\text {th }}$ annual ACM/IEEE 
International Conference on Mobile Computing and Networking, New York, NY: ACM, pp. 271-278, 1999

[2] J.P. Vasseur, A. Dunkels, Interconnecting Smart Objects with IP - the next internet, ed. Elsevier - Morgan Kaufmann publishers, 2010

[3] Kahn B. Priyantha, A. Kansal, M. Goraczko, F. Zhao, Tiny Web Services: Design and Implementation of Interoperable and Evolvable Sensor Networks, Proceedings of the $6^{\text {th }}$ ACM conference on Embedded Network Sensory Systems (sensSys '08), Raleigh, NC, USA, pp. 253-266, 2008.

[4] A. Yazar, A. Dunkels, Efficient Application Integration in IP-Based Sensor Networks, Proceedings of the ACM BuildSys 2009 Workshop, in conjunction with ACM SensSys 2009, 2009

[5] http://www.ipso-alliance.org

[6] IEEE std. 802.15.4 - 2003: Wireless Medium Access Control (MAC) and Physical Layer (PHY) specifications for Low Rate Wireless Personal Area Networks (LR-WPANs)

[7] http://standards.ieee.org/getieee802/download/802.15.4-2003.pdf

[8] http://www.zigbee.org

[9] Paradiso, J. A., Starner, T., Energy scavenging for mobile and wireless electronics, IEEE CS and IEEE ComSoc, Pervasive computing, pp. 18-27, 2005

[10] Ottmann, G., Hofmann, H., Bhatt, A., Lesieutre, G., Adaptive piezoelectric energy harvesting circuit for wireless remote power supply, IEEE Trans. Power Electron.17, pp. 669-676, 2002

[11] Roundy, J.S., Leland, E.S., Baker, J., Carleton, E., Reilly, E., Lai, E., Improving power output for vibration-based energy scavengers, Pervasive Comput. 4, pp. 28-36, 2005

[12] Atturo, C., Cianfrone, C., Ferrara, V., Fiumi, L., Fontinovo, G., Ottavi, C.M., Remote Sensing Detection Techniques for Brownfield Identification and Monitoring by GIS Tools, Proceedings of 3th international Conference Brownfields III, Ed. Brebbia, Wit Press Southampton, pp. 241-250, 2006

[13] Ferrara, V, Brownfield identification: different approaches for analysing data detected by means of remote sensing, Proceedings of international Conference Brownfields 2008, ed. Brebbia, Wit Press Southampton, pp. 4554, 2008

[14] Akyildiz, F. et al., A survey on sensor networks. IEEE Comm. Mag., 40(8), pp.102-114, 2002.

[15] Akyildiz, F. et al., A survey on wireless multimedia sensor networks, Computer Networks, Vol. 51, pp. 921-960, 2007 